\title{
Influence of sodium glucose co-transporter 2 inhibitors on fatty liver index parameters in type 2 diabetes mellitus
}

\author{
Mohammed Ali Gameil ${ }^{1 *}$ D, Mohammed Shereif Abdelgawad ${ }^{1}$ (D) Monir Hussein Bahgat ${ }^{2}$, \\ Ahmed Hassan Elsebaie ${ }^{3}$ (1) and Rehab Elsayed Marzouk ${ }^{4}$ (D)
}

\begin{abstract}
Background: Non-alcoholic fatty liver disease (NAFLD) represents a major public health challenge worldwide. It affects more than half of the patients with type 2 diabetes mellitus (T2D). It may progress to non-alcoholic steatohepatitis, cirrhosis, and carcinoma. The sodium glucose co-transporter 2 inhibitors (SGLT2 inhibitors) may improve hepatic steatosis. We aimed to estimate the effect of empagliflozin or dapagliflozin versus conventional treatment on fatty liver status in patients with concomitant T2D and NAFLD over 24 weeks.

Results: We found a significant improvement of the fatty liver index (FLI) with a significant reduction of the bodyweight, body mass index, waist circumference, ALT, AST, GGT, AST to ALT ratio, lipid profile, and lipid profile ratios in both SGLT2 inhibitors groups versus the conventional treatment group. Post hoc analysis revealed no statistically significant difference between the SGLT2 inhibitors groups (dapagliflozin versus empagliflozin).

Conclusion: SGLT2 inhibitors, empagliflozin and dapagliflozin, exert a beneficial effect on the fatty liver index of diabetic patients with NAFLD.
\end{abstract}

Keywords: NAFLD, SGLT2 inhibitors, Fatty liver index

\section{Background}

Non-alcoholic fatty liver disease (NAFLD) is the most prevalent chronic liver disease with an estimated prevalence of $25 \%$ worldwide; it affects more than $50 \%$ of patients with type 2 diabetes mellitus (T2D) [1]. NAFLD comprises a wide spectrum from steatosis to nonalcoholic steatohepatitis (NASH), fibrosis, and carcinoma [2]. Increase in fat stored as triglycerides in the liver may increase ALT and AST [3]. NAFLD is the hepatic manifestation of metabolic syndrome and is strongly linked to obesity, dyslipidaemia, and insulin resistance [4]. Gender, age, ethnicity, obesity, and T2D are risk factors for NAFL $D$ [5]. The NAFLD pathogenesis is not clearly understood, but the genetic predisposition with insulin resistance (IR)

\footnotetext{
* Correspondence: drmaligameil1979@yahoo.com

${ }^{1}$ Endocrinology Unite, Internal Medicine Department, Faculty of Medicine,

Mansoura University, Mansoura, Dakahlia, Egypt

Full list of author information is available at the end of the article
}

may lead to increase apoptosis, mitochondrial dysfunction, reactive oxygen species (ROS), lipid peroxidation of cell membranes, and pro-inflammatory genes induction [6]. This multifactorial nature of NAFLD pathogenesis represents a challenge for development of targeted therapy [7]. It is usually asymptomatic and underdiagnosed; patients may have normal liver enzymes. Liver biopsy is still the gold standard method for the diagnosis and staging [8]. Several diagnostic methods have been used for detection of fibrosis such as the NAFLD fibrosis score, the NAFLD activity score (NAS), and the fatty liver index (FLI) [9]. Liver US, CT scanning, MRI, and magnetic resonance spectroscopy (MRS) are usually used in detecting NAFLD [10]. The co-existence of NAFLD and T2D can drive adverse outcomes. The poor quality of evidence has led to scarce pharmaceutical options for NAFLD [11]. The sodium glucose co-transporter 2 inhibitors (SGLT2 inhibitors) increase glycosuria and reduce visceral adiposity and 
bodyweight, but their efficacy in patients with NAFLD has not been sufficiently investigated [12]. The aim of this study was to detect the effect of the addition of empagliflozin or dapagliflozin on the fatty liver index in patients with concomitant T2D and NAFLD as well as evaluation of the changes of the lipid profile and other estimated clinical parameters between all study groups.

\section{Methods}

A non-concurrent cohort study was conducted at the outpatient department of the authors' institution (May 2019-October 2019; 24 weeks).

Our study contained three matched groups of patients: group (1), patients administrated dapagliflozin $10 \mathrm{mg}$ daily added to their treatment, composed of 99 patients (65 male $65.7 \%$ and 34 female $34.3 \%$ ); group (2), patients administrated empagliflozin $25 \mathrm{mg}$ daily added on their treatment composed of 123 patients ( 81 male $65.9 \%$ and 42 female $34.1 \%$ ); and group (3), control group, patients administrated their conventional treatment (metformin \pm sulfonylurea) without SGLT2 inhibitors, GLP1-RA, or TZDs composed of 73 patients (44 male $60.3 \%$ and 29 female $39.7 \%$ ). Egyptian patients with type 2 DM (30-65 years old, HbA1c level above $7.5 \%$, body mass index (BMI) above $25 \mathrm{~kg} / \mathrm{m}^{2}$, and estimated glomerular filtration rate $(\mathrm{eGFR}) \geq 45 \mathrm{~mL} / \mathrm{min} / 1.73 \mathrm{~m}^{2}$ ), who attended the outpatient clinics at the authors' institution, were enrolled in this study. After signing a written informed consent, a detailed medical history and thorough physical examination with anthropometric measures were done. Blood samples were obtained in the morning following a 12-h overnight fast for assessment of HbA1c, lipid profiles, liver function tests, renal function tests, and thyroid profile. The results were compared before and after 24 weeks. Hypertension was diagnosed in patients with $\mathrm{SBP} \geq 130 \mathrm{mmHg}$ and/or DBP $\geq 85 \mathrm{mmHg}$ or in those taking antihypertensive medication. Dyslipidaemia was identified in patients with serum triglyceride (TG) levels $\geq 150 \mathrm{mg} / \mathrm{dL}$ and/or high-density lipoprotein cholesterol (HDL-C) levels $<40 \mathrm{mg} / \mathrm{dL}$. The extent of the fatty liver was estimated using the fatty liver index (FLI), comprising BMI, waist circumference (WC in $\mathrm{cm}$ ), gamma-glutamyl transferase (GGT, in IU/L), and triglycerides (TG, in $\mathrm{mg} / \mathrm{dL}$ ). This index was calculated using the following equation: FLI $=\{(\exp (0.953 \times \log (\mathrm{TG})+0.139 \times$ $\mathrm{BMI}+0.718 \times \log (\mathrm{GGT})+0.053 \times \mathrm{WC}-15.745) / 1+\exp$ $(0.953 \times \log (\mathrm{TG})+0.139 \times \mathrm{BMI}+0.718 \times \log (\mathrm{GGT})+$ $0.053 \times \mathrm{WC}-15.745)\} \times 100$. FLI $<30$ can be used to rule out hepatic steatosis [13].

We excluded patients with other causes of chronic liver diseases like viral hepatitis, autoimmune hepatitis, alcoholic consumption, drug-induced liver injury, biliary tract disease, decompensated liver cirrhosis, hepatocellular carcinoma, history of diabetic ketoacidosis, co-administration of medications that may affect liver function tests (such as NSAIDS, statin, and TZDs), and the presence of severe comorbidities such as heart, respiratory, and/or renal dysfunction.

Participant's visits were every 2 months to ensure that they were committed to the medications. Adverse events were recorded on each visit, and the severity was assessed according to the Common Terminology Criteria for Adverse Events Version 4.0.

\section{Statistical analysis}

Data were entered and analysed using the IBM-SPSS software (IBM Corp. Released 2017. IBM SPSS Statistics for Windows, Version 25.0. Armonk, NY: IBM Corp.). Qualitative data were expressed as frequency (percentage) and compared by the chi-square test. Quantitative data were expressed as mean \pm standard deviation (SD) due to its normality (Shapiro-Wilk's test, $p>0.050$ ) and no significant outliers (extreme values) as examined by inspecting the boxplots. Quantitative data were compared between the three groups using one-way ANOVA for age and DM duration and using one-way ANCOVA for all other clinical and laboratory parameters (measured over two-time points) where the 6-month reading (dependent variable) was compared between the three groups after adjustment for the initial reading at enrolment (covariate). In both tests' results, partial eta-squared $\left(\eta^{2}\right)$ was an estimate of the effect size. For any of the used tests, results were considered as statistically significant if $p$ value $\leq 0.050$.

\section{Results}

We found a statistically significant difference in all studied parameters between the three groups ( $p$ value $<0.05$ ) except for HDL-C that was of similar values between the three groups ( $p$ value $=0.863$ ) as shown in Tables 1 and 2 and Figs. 1, 2, and 3 showed a significant improvement of the fatty liver index with a significant reduction of each of weight, BMI, waist circumference, ALT, AST, $\delta$ glutamyl transferase, and AST to ALT ratio in the two groups who are administrated empagliflozin and dapagliflozin compared to the conventional treatment group ( $p$ value $<0.001$ ). There was a significant improvement of the lipid profile (TG, total cholesterol, LDL-C, VLDL-C, TC to HDL-C ratio, TG to HDL-C and LDL to HDL-C ratio) in the patients who are administrated SGLT2 inhibitors in comparison to the control group ( $p$ value $<0.05$ ) as shown in Table 2 and Fig. 4. After post hoc analysis, we did not find any statistically significant difference between the SGLT2 inhibitors groups (dapagliflozin versus empagliflozin) in all the studied parameters. However, there was a statistically significant difference in all studied parameters between both SGLT2 inhibitors 


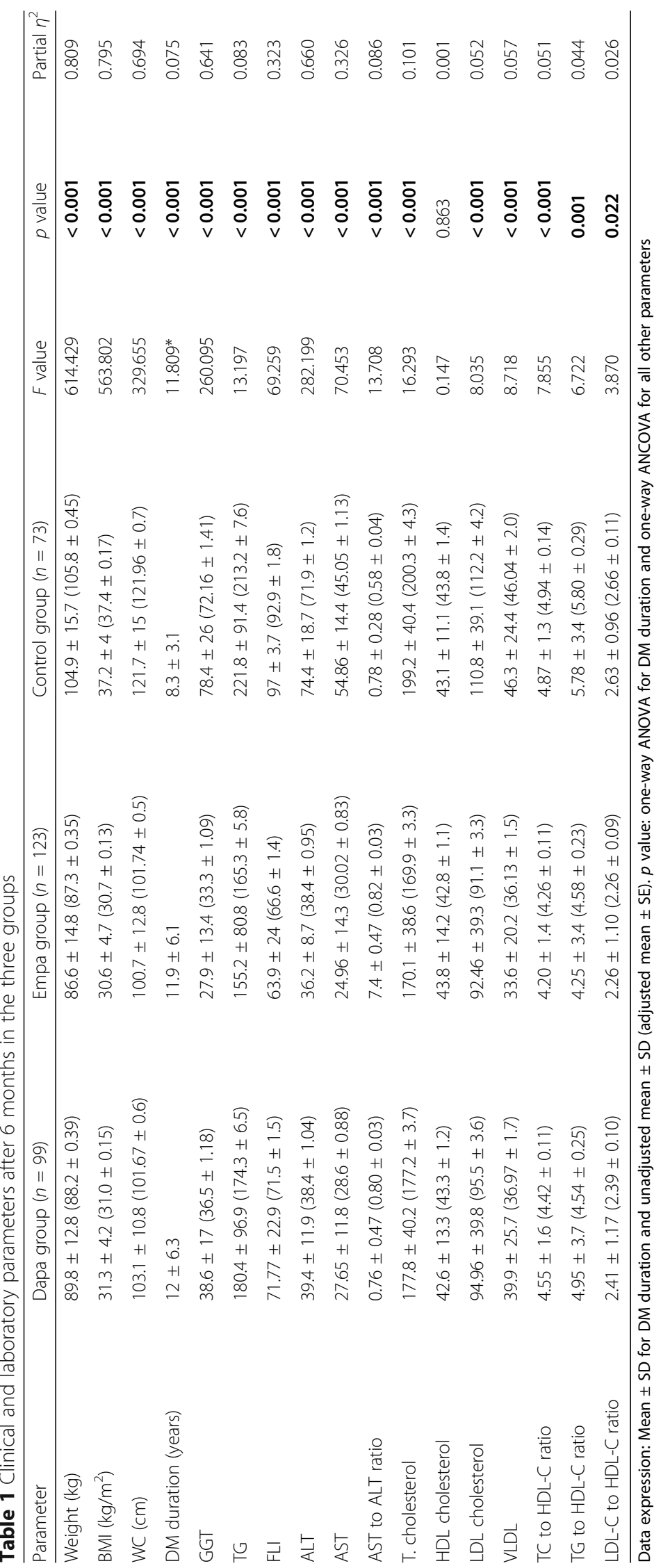


Table 2 Post hoc analysis with a Bonferroni adjustment for study parameters

\begin{tabular}{|c|c|c|c|c|}
\hline Parameter & Pair & Mean difference & $95 \% \mathrm{Cl}$ & $p$ value \\
\hline \multirow[t]{3}{*}{ Weight (kg) } & 1 vs 2 & 0.951 & -0.297 to 2.198 & 0.202 \\
\hline & 1 vs 3 & -17.56 & -18.98 to -16.13 & $<0.001$ \\
\hline & 2 vs 3 & -18.509 & -19.87 to -17.15 & $<0.001$ \\
\hline \multirow[t]{3}{*}{ BMI $\left(\mathrm{kg} / \mathrm{m}^{2}\right)$} & 1 vs 2 & 0.313 & -0.156 to 0.782 & 0.327 \\
\hline & 1 vs 3 & -6.351 & -6.887 to -5.815 & $<0.001$ \\
\hline & 2 vs 3 & -6.664 & -7.177 to -6.151 & $<0.001$ \\
\hline \multirow[t]{3}{*}{ WC (cm) } & 1 vs 2 & -0.078 & -1.987 to 1.831 & 1.000 \\
\hline & 1 vs 3 & -20.290 & -22.46 to -18.12 & $<0.001$ \\
\hline & 2 vs 3 & -20.212 & -22.29 to -18.13 & $<0.001$ \\
\hline \multirow[t]{3}{*}{ DM duration (years) } & 1 vs 2 & 0.1321 & -1.843 to 2.107 & 0.986 \\
\hline & 1 vs 3 & 3.7224 & 1.996 to 5.449 & $<0.001$ \\
\hline & 2 vs 3 & 3.5904 & 2.045 to 5.136 & $<0.001$ \\
\hline \multirow[t]{3}{*}{ GGT } & 1 vs 2 & 3.243 & -0.680 to 7.165 & 0.142 \\
\hline & 1 vs 3 & -35.626 & -39.98 to -31.27 & $<0.001$ \\
\hline & 2 vs 3 & -38.868 & -43.31 to -34.42 & $<0.001$ \\
\hline \multirow[t]{3}{*}{ TG } & 1 vs 2 & 9.026 & -12.05 to 30.1 & 0.910 \\
\hline & 1 vs 3 & -38.946 & -62.9 to -15.03 & $<0.001$ \\
\hline & 2 vs 3 & -47.973 & -71.05 to -24.9 & $<0.001$ \\
\hline \multirow[t]{3}{*}{ FLI } & 1 vs 2 & 4.883 & -0.081 to 9.846 & 0.056 \\
\hline & 1 vs 3 & -21.354 & -27.02 to -15.69 & $<0.001$ \\
\hline & 2 vs 3 & -26.237 & -31.75 to -20.73 & $<0.001$ \\
\hline \multirow[t]{3}{*}{ ALT } & 1 vs 2 & -0.076 & -3.484 to 3.333 & 1.000 \\
\hline & 1 vs 3 & -33.580 & -37.416 to -29.744 & $<0.001$ \\
\hline & 2 vs 3 & -33.505 & -37.276 to -29.734 & $<0.001$ \\
\hline \multirow[t]{3}{*}{ AST } & 1 vs 2 & -1.420 & -4.300 to 1.460 & 0.709 \\
\hline & 1 vs 3 & -16.455 & -19.940 to -12.969 & $<0.001$ \\
\hline & 2 vs 3 & -15.035 & -18.608 to -11.462 & $<0.001$ \\
\hline \multirow[t]{3}{*}{ AST to ALT ratio } & 1 vs 2 & -0.021 & -0.124 to 0.081 & 1.000 \\
\hline & 1 vs 3 & 0.226 & 0.104 to 0.349 & $<0.001$ \\
\hline & 2 vs 3 & 0.248 & 0.128 to 0.367 & $<0.001$ \\
\hline \multirow[t]{3}{*}{ T. cholesterol } & 1 vs 2 & 7.347 & -4.500 to 19.193 & 0.409 \\
\hline & 1 vs 3 & -23.017 & -36.540 to -9.494 & $<0.001$ \\
\hline & 2 vs 3 & -30.364 & -43.330 to -17.397 & $<0.001$ \\
\hline \multirow[t]{3}{*}{ LDL cholesterol } & 1 vs 2 & 4.373 & -7.418 to 16.164 & 1.000 \\
\hline & 1 vs 3 & -16.712 & -30.170 to -3.255 & 0.009 \\
\hline & 2 vs 3 & -21.086 & -34.001 to -8.170 & $<0.001$ \\
\hline \multirow[t]{3}{*}{ VLDL } & 1 vs 2 & 0.837 & -4.747 to 6.421 & 1.000 \\
\hline & 1 vs 3 & -9.071 & -15.393 to -2.749 & 0.002 \\
\hline & 2 vs 3 & -9.908 & -15.966 to -3.850 & $<0.001$ \\
\hline \multirow[t]{3}{*}{ TC to HDL-C ratio } & 1 vs 2 & 0.159 & -0.226 to 0.544 & 0.964 \\
\hline & 1 vs 3 & -0.522 & -0.961 to -0.083 & 0.014 \\
\hline & 2 vs 3 & -0.681 & -1.100 to -0.261 & $<0.001$ \\
\hline \multirow[t]{2}{*}{ TG to HDL-C ratio } & 1 vs 2 & -0.032 & -0.850 to 0.787 & 1.000 \\
\hline & 1 vs 3 & -1.252 & -2.181 to -0.323 & 0.004 \\
\hline
\end{tabular}


Table 2 Post hoc analysis with a Bonferroni adjustment for study parameters (Continued)

\begin{tabular}{lllll}
\hline Parameter & Pair & Mean difference & $95 \% \mathrm{Cl}$ & $p$ value \\
\hline \multirow{3}{*}{ LDL-C to HDL-C ratio } & 2 vs 3 & -1.220 & -2.109 to -0.331 & $\mathbf{0 . 0 0 3}$ \\
& 1 vs 2 & 0.130 & -0.184 to 0.443 & 0.959 \\
& 1 vs 3 & -0.266 & -0.624 to 0.092 & 0.224 \\
& 2 vs 3 & -0.396 & -0.739 to -0.053 & $\mathbf{0 . 0 1 7}$ \\
\hline
\end{tabular}

$1=$ Dapa, 2 = Empa, and 3 = control

groups versus the control group $(p$ value $<0.05)$ as shown in Table 2.

\section{Discussion}

In our study, after the use of SGLT2 inhibitors, there was a significant improvement of the fatty liver index as well as a significant reduction of each of the bodyweight, BMI, waist circumference, ALT, AST, $\delta$ glutamyl transferase, and AST to ALT ratio in the empagliflozin and dapagliflozin groups versus the conventional treatment group. After post hoc analysis, we did not find any statistically significant difference between the SGLT2 inhibitors groups (dapagliflozin versus empagliflozin) in all the studied parameters. However, there was a statistically significant difference in all studied parameters between both SGLT2 inhibitors groups versus the conventional treatment group.

Our results in the empagliflozin group agreed with Kuchay et al. [14] who studied the use of empagliflozin for 20 weeks in T2D patients and showed a significant reduction in the liver fat mass evaluated by MRI proton density fat fraction (MRI-PDFF), as well as a significant reduction in serum ALT level.

In our study, the significant reduction of ALT, AST, GGT, bodyweight, and HBA1c in the empagliflozin group agreed with Sattar et al. [15] who found a significant improvement of ALT, HbA1c, and bodyweight with sub-analysis of the EMPA-REG trial.
Our results agreed with Jojima et al. [16] who studied the use of empagliflozin and combination of empagliflozin and linagliptin versus placebo and found empagliflozin as a monotherapy or a dual therapy reduced the severity of NASH in NASH mouse models.

The effects of empagliflozin and canagliflozin in patients with T2DM and NASH were evaluated by Lai et al. [17] who found a significant improvement in the histology, ALT, AST, FIB-4 index, and other metabolic parameters that coincided with our results despite variations in the study population, duration, and design.

Our results in the dapagliflozin group agreed with Wang et al. [18] who found an improvement in aminotransferases, hepatic steatosis, and fibrosis in obese mice after dapagliflozin use. Also, Tang et al. [19] found the treatment with dapagliflozin improved hepatic damage markers like myeloperoxidase (MPO) and oxidative stress in genetic murine models of obesity and diabetes.

Also, our findings were in agreement with Tobita et al. [20] who used dapagliflozin in patients with biopsyproven NASH with type $2 \mathrm{DM}$ for 6 months that led to a significant improvement of liver enzymes, obesity, and glycaemic control.

Our results were consistent with Lee et al. [21] and Shimizu et al. [22] who demonstrated a significant reduction in ALT, GGT, and liver stiffness, which suggested that dapagliflozin can significantly attenuate severe liver fibrosis. Also, Eriksson et al. [23] found the

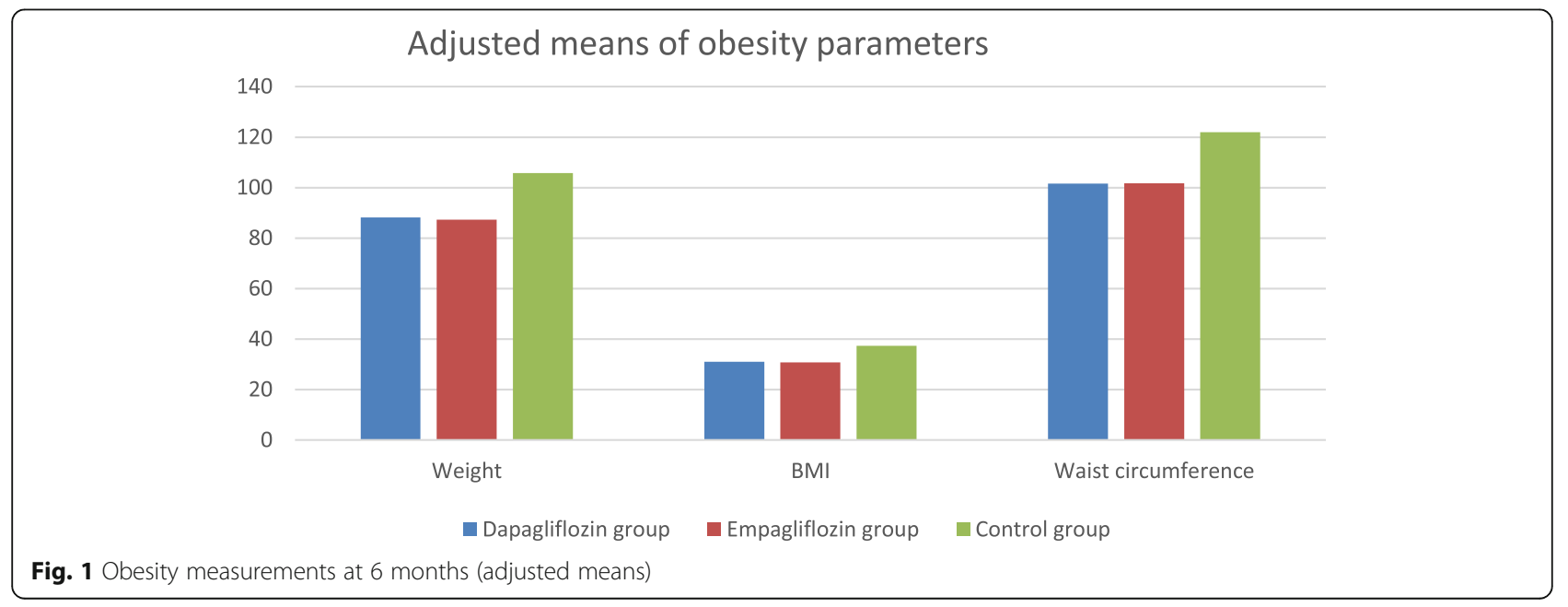




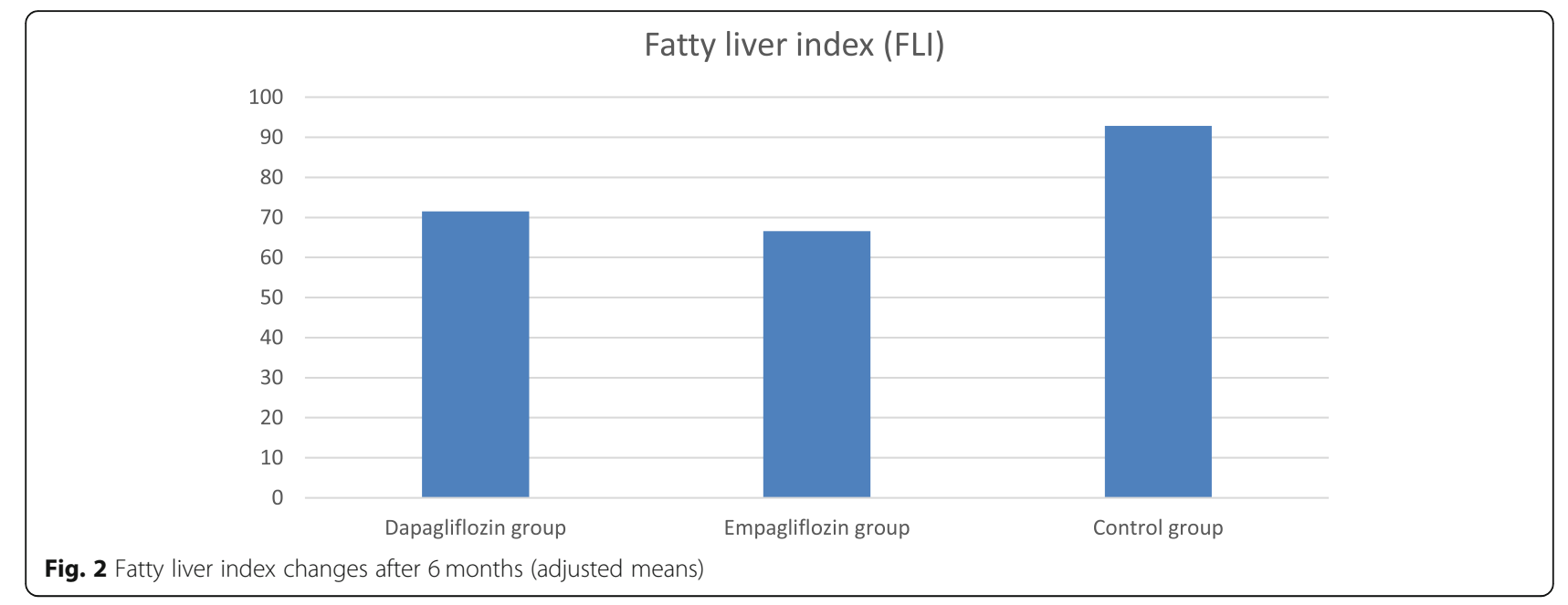

combination of dapagliflozin and n-3 carboxylic acid increased adiponectin and zinc-A2-glycoprotein secretion improving insulin resistance and hepatic steatosis.

Jojima et al. [16] attributed the hepatic steatosis improvement with SGLT2 inhibitors to the decrease of collagen deposition and the downregulation of inflammatory cytokines in the liver. However, Henith et al. [24] and Shibuya et al. [25] attributed the beneficial effects of SGLT-2 inhibitors on liver enzymes, liver fat, fibrosis, and various metabolic parameters to the efficient improvement of glycaemic control and insulin resistance. Mohammad et al. [26] explained the beneficial effects of SGLT-2 inhibitors in amelioration

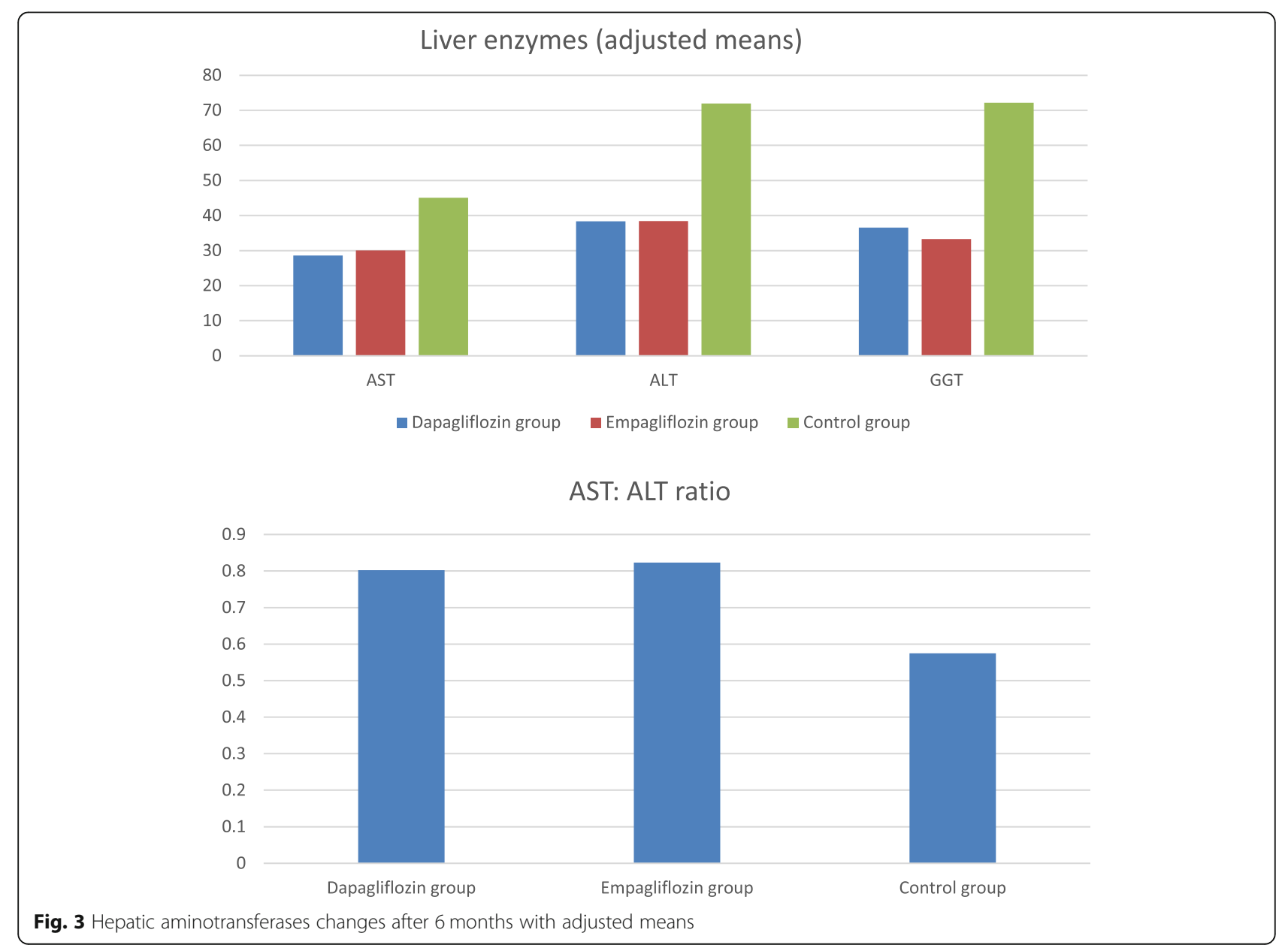




\section{Lipid profile (adjusted means)}

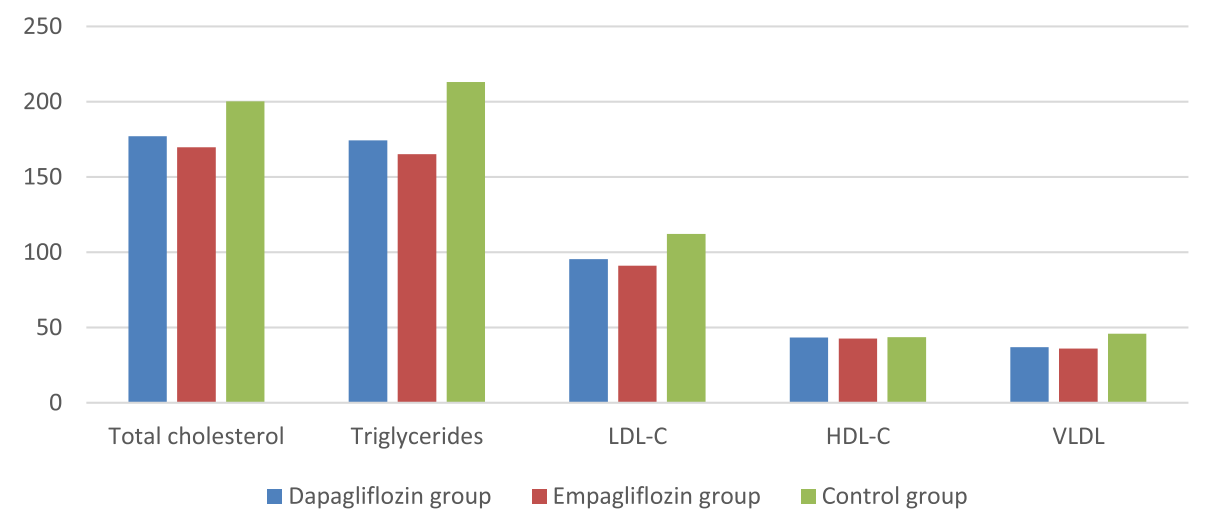

Lipid profile ratios (adjusted means)

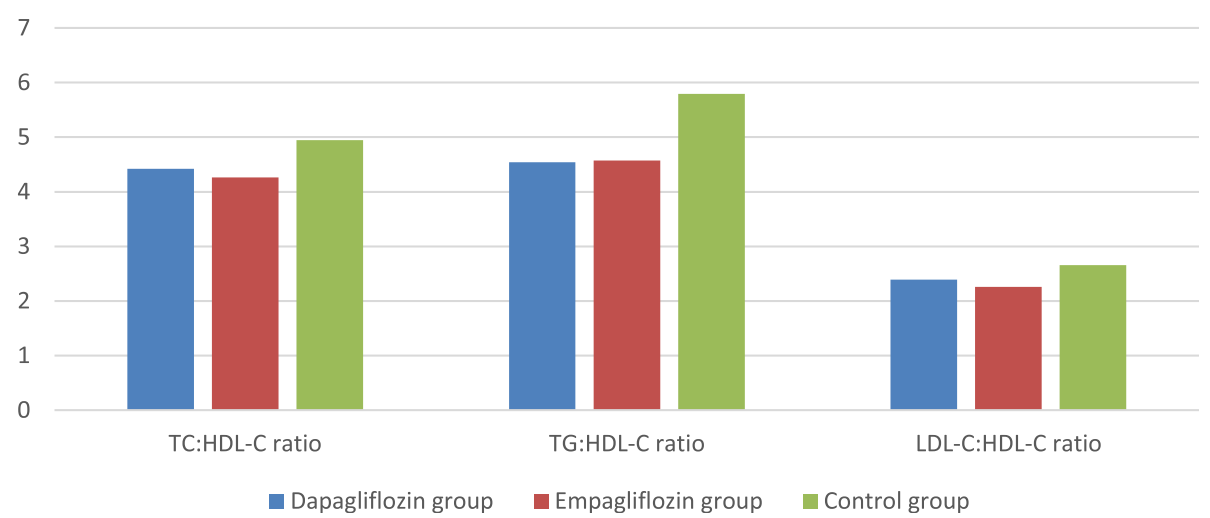

Fig. 4 Lipid profile after 6 months (adjusted means)

of insulin resistance via decreasing glucotoxicity, regulating macrophage recruitment, increasing the anti-inflammatory cytokines, and inducing the expression of thermogenin which promotes $\beta$-oxidation of fatty acids with lipid profile improvement.

We found a significant improvement of the lipid profile (TG, total cholesterol, LDL-C, VLDL-C, TC to HDL$\mathrm{C}$ ratio, TG to $\mathrm{HDL}-\mathrm{C}$ ratio, and $\mathrm{LDL}$ to $\mathrm{HDL}-\mathrm{C}$ ratio) in the SGLT2 inhibitors groups in comparison to the control group that may be attributed to the improved glycaemic control and weight loss. This significant reduction of serum TG levels and the TG/HDL-C ratio agreed with Hayashi et al. [27] who studied the effect of dapagliflozin on lipid profile and found the decrease in the TG/HDL-C ratio may lower small dense LDL-C levels, subsequently decreasing the incidence of cardiovascular events.

There was a significant bodyweight reduction in the SGLT2 inhibitor groups which is in accordance with Ferrannini et al. [28] who attributed this effect to the significant decrease of fat mass through increasing lipid utilization more than carbohydrates.
The major point of strength in our study was the use of two different SGLT-2 inhibitor molecules (empagliflozin and dapagliflozin) in 2 comparable arms in patients with type 2 diabetes and NAFLD. However, our limitations were the ethnicity issue and the single-centre study. Longterm prospective studies, preferably a randomized controlled trial, applying invasive histopathology sampling are warranted in the future.

\section{Conclusion}

The patients with concomitant T2D and NAFLD can gain many clinical and metabolic benefits with the administration of SGLT-2 inhibitors mainly through the improvement of the hepatic steatosis, dyslipidaemia, glycaemic control, and weight loss.

\section{Abbreviations}

NAFLD: Non-alcoholic fatty liver disease; T2D: Type 2 diabetes mellitus; NASH: Non-alcoholic steatohepatitis; TZDs: Thiazolidinediones; SGLT2 inhibitors: Sodium glucose co-transporter 2 inhibitors; BMl: Body mass index; FLI: Fatty liver index; FIB-4: Fibrosis-4 index; IR: Insulin resistance; BW: Bodyweight; BMI: Body mass index; WC: Waist circumference; TG: Triglycerides; TC: Total cholesterol; TG: Triglyceride; HDL-C: High-density 
lipoprotein cholesterol; LDL-C: Low-density lipoprotein cholesterol; VLDLC: Very low-density lipoprotein cholesterol

\section{Acknowledgements}

Not applicable.

\section{Authors' contributions}

Mohammed Ali Gameil contributed to the conception, study design, and literature review, analysed and interpreted the patient data regarding clinical and laboratory findings before and after treatment course, and shared in the manuscript writing. Mohammed Shereif Abd El Gawad was the major contributor in manuscript draft writing and contributed to the critical revision of the results and writing of the final manuscript version. Monir Hussein Bahgat was the major contributor to the data analysis and statistical work and shared in the critical revision and editing of the final manuscript. Ahmed Hassan Elsebaie and Rehab Elsayed Marzouk were the major contributors in laboratory data revision and analysis with critical revision of the manuscript draft. All authors read and approved the final manuscript.

\section{Funding}

By authors

\section{Availability of data and materials}

The datasets used and/or analysed during the current study are available from the corresponding author on reasonable request.

\section{Ethics approval and consent to participate}

This study was approved by the Institutional Review Board for Clinical Research committee of Mansoura University with approval number No.R.20.04.804. All procedures performed in studies involving human participants were in accordance with the ethical standards of the institutional research committee and with the 1964 Helsinki Declaration and its later amendments or comparable ethical standards.

Written informed consent was approved by the Institutional Review Board for Clinical Research committee of Mansoura University and obtained from all participants.

\section{Consent for publication}

Obtained from all participants.

\section{Competing interests}

The authors declare that they have no competing interests.

\begin{abstract}
Author details
${ }^{1}$ Endocrinology Unite, Internal Medicine Department, Faculty of Medicine Mansoura University, Mansoura, Dakahlia, Egypt. ${ }^{2}$ Gastroenterology Unite, Internal Medicine Department, Faculty of Medicine, Mansoura University, Mansoura, Dakahlia, Egypt. ${ }^{3}$ Clinical Pathology Department, Faculty of Medicine, Mansoura University, Mansoura, Dakahlia, Egypt. ${ }^{4}$ Medical Biochemistry Department, Faculty of Medicine, Mansoura University, Mansoura, Dakahlia, Egypt.
\end{abstract}

\section{Received: 1 June 2020 Accepted: 27 July 2020}

Published online: 18 August 2020

\section{References}

1. Dai W, Ye L, Liu A, Wen SW, Deng J, Wu X et al (2017) Prevalence of nonalcoholic fatty liver disease in patients with type 2 diabetes mellitus: a meta-analysis. Medicine (Baltimore) 96(39):e8179

2. Chalasani N, Younossi Z, Lavine JE, Diehl AM, Brunt EM, Cusi K et al (2012) The diagnosis and management of non-alcoholic fatty liver disease: practice Guideline by the American Association for the Study of Liver Diseases, American College of Gastroenterology, and the American Gastroenterological Association. Hepatology 55(6):2005-2023

3. Sattar N, Forrest E, Preiss D (2014) Non-alcoholic fatty liver disease. BMJ 349: g4596. https://doi.org/10.1136/bmj.g4596

4. Yki-Järvinen $H$ (2014) Non-alcoholic fatty liver disease as a cause and a consequence of metabolic syndrome. Lancet Diabetes Endocrinol 2(11): 901-910

5. Rich NE, Oji S, Mufti AR, Browning JD, Parikh ND, Odewole M et al (2018) Racial and ethnic disparities in nonalcoholic fatty liver disease prevalence, severity, and outcomes in the United States: a systematic review and metaanalysis. Clin Gastroenterol Hepatol 16(2):198-210.e2

6. Wolfs MG, Gruben N, Rensen SS, Verdam FJ, Greve JW, Driessen A et al (2015) Determining the association between adipokine expression in multiple tissues and phenotypic features of non-alcoholic fatty liver disease in obesity. Nutr Diabetes 5:e146

7. Petersen KF, Dufour S, Befroy D, Lehrke M, Hendler RE, Shulman Gl (2005) Reversal of nonalcoholic hepatic steatosis, hepatic insulin resistance, and hyperglycemia by moderate weight reduction in patients with type 2 diabetes. Diabetes 54(3):603-608

8. Sanyal AJ, Brunt EM, Kleiner DE, Kowdley KV, Chalasani N, Lavine JE et al (2011) Endpoints and clinical trial design for nonalcoholic steatohepatitis. Hepatology 54(1):344-353

9. Angulo P, Hui JM, Marchesini G, Bugianesi E, George J, Farrell GC et al (2007) The NAFLD fibrosis score: a noninvasive system that identifies liver fibrosis in patients with NAFLD. Hepatology 45(4):846-854

10. Noureddin M, Lam J, Peterson MR, Middleton M, Hamilton G, Le TA et al (2013) Utility of magnetic resonance imaging versus histology for quantifying changes in liver fat in nonalcoholic fatty liver disease trials. Hepatology 58(6):1930-1940

11. Targher G, Bertolini L, Rodella S, Tessari R, Zenari L, Lippi G (2007) Nonalcoholic fatty liver disease is independently associated with an increased incidence of cardiovascular events in type 2 diabetic patients. Diabetes Care 30:2119-2121

12. Arase $Y$, Shiraishi K, Anzai K, Sato H, Teramura E, Tsuruya K et al (2019) Effect of sodium glucose co-transporter 2 inhibitors on liver fat mass and body composition in patients with nonalcoholic fatty liver disease and type 2 diabetes mellitus. Clin Drug Investig 39(7):631-641. Published online 2019 Apr 16. https://doi.org/10.1007/s40261-019-00785-6

13. Bedogni G, Bellentani S, Miglioli L, Masutti F, Passalacqua M et al (2006) The Fatty Liver Index: a simple and accurate predictor of hepatic steatosis in the general population. BMC Gastroenterol 6:33

14. Kuchay MS, Krishan S, Mishra SK, Farooqui KJ, Singh MK, Wasir JS et al (2018) Effect of empagliflozin on liver fat in patients with type 2 diabetes and nonalcoholic fatty liver disease: a randomized controlled trial (E-LIFT Trial). Diabetes Care 41(8):1801-1808

15. Sattar N, Fitchett D, Hantel S, George JT, Zinman B (2018) Empagliflozin is associated with improvements in liver enzymes potentially consistent with reductions in liver fat: results from randomised trials including the EMPAREG OUTCOME ${ }^{\oplus}$ trial. Diabetologia 61(10):2155-2163

16. Jojima T, Tomotsune T, lijima T, Akimoto K, Suzuki K, Aso Y (2016) Empagliflozin (an SGLT2 inhibitor), alone or in combination with linagliptin (a DPP-4 inhibitor), prevents steatohepatitis in a novel mouse model of non-alcoholic steatohepatitis and diabetes. Diabetol Metab Syndr 8:45

17. Lai LL, Vethakkan SR, Nik Mustapha NR, Mahadeva S, Chan WK (2020) Empagliflozin for the treatment of nonalcoholic steatohepatitis in patients with type 2 diabetes mellitus. Dig Dis Sci 65(2):623-631. https://doi.org/10. 1007/s10620-019-5477-1

18. Wang D, Luo Y, Wang X, Orlicky DJ, Myakala K, Yang P et al (2018) The sodium-glucose cotransporter 2 inhibitor dapagliflozin prevents renal and liver disease in western diet induced obesity mice. Int J Mol Sci 19(1). https://doi.org/10.3390/ijms19010137

19. Tang L, Wu Y, Tian M, Sjöström CD, Johansson U, Peng XR et al (2017) Dapagliflozin slows the progression of the renal and liver fibrosis associated with type 2 diabetes. Am J Physiol Endocrinol Metab 313(5):E563-E576

20. Tobita H, Sato S, Miyake T, Ishihara S, Kinoshita Y (2017) Effects of dapagliflozin on body composition and liver tests in patients with nonalcoholic steatohepatitis associated with type 2 diabetes mellitus: a prospective, open-label, uncontrolled study. Curr Ther Res Clin Exp 87:13-19

21. Lee PCH, Gu Y, Yeung MY, Fong CHY, Woo YC, Chow WS et al (2018) Dapagliflozin and empagliflozin ameliorate hepatic dysfunction among Chinese subjects with diabetes in part through glycemic improvement: a single-center, retrospective, observational study. Diabetes Ther 9(1):285-295

22. Shimizu M, Suzuki K, Kato K, Jojima T, lijima T, Murohisa T et al (2019) Evaluation of the effects of dapagliflozin, a sodium-glucose co-transporter-2 inhibitor, on hepatic steatosis and fibrosis using transient elastography in patients with type 2 diabetes and non-alcoholic fatty liver disease. Diabetes Obes Metab 21(2):285-292

23. Eriksson JW, Lundkvist $P$, Jansson PA, Johansson $L$, Kvarnström M, Moris L et al (2018) Effects of dapagliflozin and n-3 carboxylic acids on non- 
alcoholic fatty liver disease in people with type 2 diabetes: a double-blind randomised placebo-controlled study. Diabetologia 61(9):1923-1934

24. Raj H, Durgia H, Palui R, Kamalanathan S, Selvarajan S, Kar SS et al (2019) SGLT-2 inhibitors in non-alcoholic fatty liver disease patients with type 2 diabetes mellitus: a systematic review. World J Diabetes 10(2):114-132. Published, online 2019 Feb 15. https://doi.org/10.4239/wjd.v10.i2.114

25. Shibuya T, Fushimi N, Kawai M, Yoshida Y, Hachiya H, Ito S et al (2018) Luseogliflozin improves liver fat deposition compared to metformin in type 2 diabetes patients with non-alcoholic fatty liver disease: a prospective randomized controlled pilot study. Diabetes Obes Metab 20:438-442

26. Mohammad SH, Fadhil NN, Mahmood MD (2018) Effects of metformin and dapagliflozin on glycemic indices and HOMA-IR in type 2 diabetes mellitus patients. Int J Pharm Bio Sci 8:66-73

27. Hayashi T, Fukui T, Nakanishi N, Yamamoto S, Tomoyasu M, Osamura A et al (2017) Dapagliflozin decreases small dense low-density lipoprotein-cholesterol and increases high-density lipoprotein 2-cholesterol in patients with type 2 diabetes: comparison with sitagliptin. Cardiovasc Diabetol 16(1):8

28. Ferrannini E, Baldi S, Frascerra S, Astiarraga B, Heise T, Bizzotto R et al (2016) Shift to fatty substrate utilization in response to sodium-glucose cotransporter 2 inhibition in subjects without diabetes and patients with type 2 diabetes. Diabetes 65(5):1190-1195

\section{Publisher's Note}

Springer Nature remains neutral with regard to jurisdictional claims in published maps and institutional affiliations.

\section{Submit your manuscript to a SpringerOpen ${ }^{\circ}$ journal and benefit from:}

- Convenient online submission

- Rigorous peer review

- Open access: articles freely available online

High visibility within the field

- Retaining the copyright to your article

Submit your next manuscript at $\boldsymbol{\nabla}$ springeropen.com 